\title{
NEW CENTRAL CONFIGURATIONS OF THE $(n+1)$-BODY PROBLEM
}

\author{
ANTONIO CARLOS FERNANDES ${ }^{A}$, BRAULIO AUGUSTO GARCIA ${ }^{A}$, JAUME $_{\text {LLIBRE }}{ }^{B}$ \\ AND LUIS FERNANDO MELLO ${ }^{A}$
}

\begin{abstract}
In this article we study central configurations of the $(n+1)$-body problem. For the planar $(n+1)$-body problem we study central configurations performed by $n \geq 2$ bodies with equal masses at the vertices of a regular $n$-gon and one body with null mass. We also study spatial central configurations considering $n$ bodies with equal masses at the vertices of a regular polyhedron and one body with null mass.
\end{abstract}

\section{IntRodUCTION AND STATEMENT OF THE MAIN RESULTS}

The $N$-body problem consists in study the motion of $N$ pointlike masses in $\mathbb{R}^{2}$ or $\mathbb{R}^{3}$, interacting among themselves through no other forces than their mutual gravitational attraction according to Newton's gravitational law.

The equations of motion of the $N$-body problem are

$$
m_{i} \ddot{r}_{i}=-\sum_{\substack{j=1 \\ j \neq i}}^{N} \frac{m_{j} m_{i}}{r_{i j}^{3}}\left(r_{i}-r_{j}\right),
$$

for $i=1, \ldots, N$. Here we have chosen the units of length in order that the gravitational constant be equal to one, $r_{k} \in \mathbb{R}^{2}$ or $\mathbb{R}^{3}$ is the position vector of the punctual mass $m_{k}$ in an inertial system, and $r_{j k}=\left|r_{j}-r_{k}\right|$ is the Euclidean distance between $m_{j}$ and $m_{k}$.

Since the general solution of the $N$-body problem cannot be given, great importance has been attached from the very beginning to the search for particular solutions where the $N$ mass points fulfilled certain initial conditions. Thus a homographic solution of the $N$-body problem is a solution such that the configuration formed by the $N$-bodies at the instant $t$ remains similar to itself as $t$ varies.

Two configurations are similar if we can pass from one to the other doing a dilation and/or a rotation.

The first three homographic solutions where found in 1767 by Euler [8] in the 3-body problem. For these three solutions the configuration of the 3 bodies is collinear.

In 1772 Lagrange [14] found two additional homographic solutions in the 3-body problem. Now the configuration formed by the 3 bodies is an equilateral triangle.

2010 Mathematics Subject Classification. Primary 70F10, 70F15, 37N05.

Key words and phrases. Central configuration, $(n+1)$-body problem, celestial mechanics.

L.F. Mello is the corresponding author. 
At a given instant $t=t_{0}$ the configuration of the $N$-bodies is central if the gravitational acceleration $\ddot{r}_{i}$ acting on every mass point $m_{i}$ is proportional with the same constant of proportionality to its position $r_{i}$; that is

$$
\ddot{r}_{i}=-\sum_{\substack{j=1 \\ j \neq i}}^{N} \frac{m_{j}}{r_{i j}^{3}}\left(r_{i}-r_{j}\right)=\lambda r_{i}
$$

for $i=1, \ldots, N$. We refer to equations (1) as the equations of central configurations.

Pizzetti proved that the configuration of the $N$ bodies in a homographic solution is central at any instant of time, see [24].

It is important to note that homographic solutions with rotation and eventually with a dilation only exist for planar central configurations. For spatial central configurations all the homographic solutions only have dilation, see for instance [24].

If we have a central configuration, a dilation and a rotation of it, provide another central configuration. We say that two central configurations are related if we can pass from one to the other through a dilation and a rotation. This relation is an equivalence. In what follows we will talk about the classes of central configurations defined by this equivalence relation.

Central configurations of the $N$-body problem are important because:

(1) They allow to compute all the homographic solutions.

(2) If the $N$ bodies are going to a simultaneous collision, then the particles tend to a central configuration.

(3) If the $N$ bodies are going simultaneously at infinity in parabolic motion (i.e. the radial velocity of each particle tends to zero as the particle tends to infinity), then the particles tend to a central configuration.

(4) There is a relation between central configurations and the bifurcations of the hypersurfaces of constant energy and angular momentum.

(5) Central configurations provides good places for the observation in the solar system, for instance, SOHO project.

(6) $\ldots$

For more information about central configurations see for a classical introduction the books of Wintner [24] and Hagihara [11], and for a modern one see, for instance, the papers of Albouy and Chenciner [1], Albouy and Kaloshin [2], Gómez, Llibre, Martínez and Simó [9, 10], Hampton and Moeckel [12], Meyer [15], Moeckel [16], Palmore [17], Saari [19], Schmidt [22], Smale [23], Xia [25], ...

In this article we are interested in the following kind of central configurations: Consider $n \geq 2$ bodies with positive equal masses at the vertices of either a regular $n$-gon in the planar case or a regular polyhedron in the spatial case. Where can a body with null mass be located in order to have a new central configuration?

The above problem is part of the well known restricted $(n+1)$-body problem. Of course, it has at least one solution for both planar and spatial cases: the body with null mass at the center of the regular $n$-gon or of the regular polyhedron. 


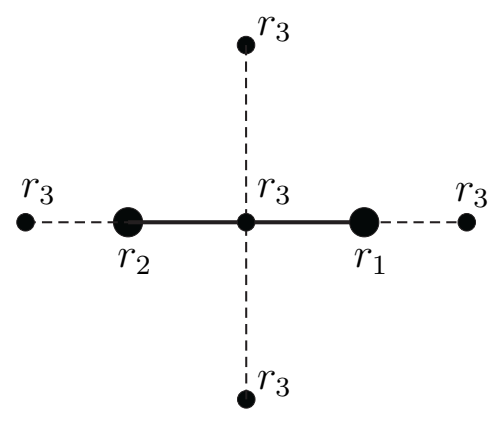

(a)

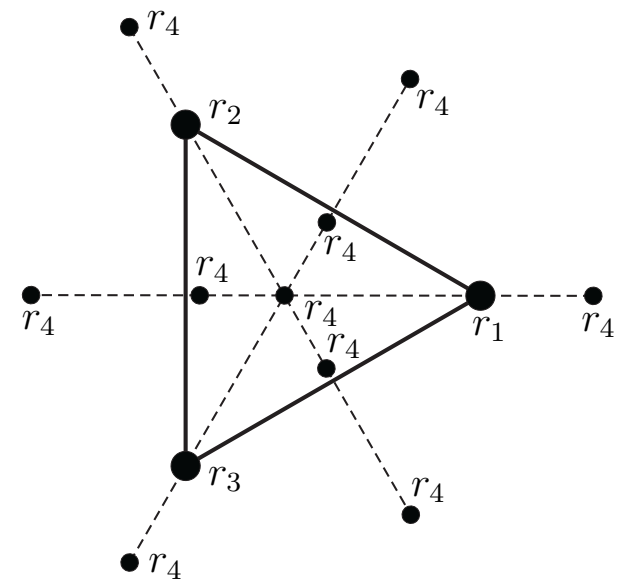

(b)

Figure 1. (a) Two bodies with equal masses and position vectors $r_{1}, r_{2}$ at the endpoints of a segment and the 5 possible positions $r_{3}$ for the third body with null mass. (b) Three bodies with equal masses and position vectors $r_{1}, r_{2}, r_{3}$ at the vertices of an equilateral triangle and the 10 possible positions $r_{4}$ for the fourth body with null mass.

To the best of our knowledge, the case $n=3$ was firstly studied by Pedersen [18] and after by Arenstorf [3] who gave the following solution: there are 10 possible positions to the body with null mass being one at the center of the equilateral triangle and 3 on each of the 3 lines of symmetry of the equilateral triangle (see Figure 1 (b)). This last result was extended by Bang and Elmabsout [4], who proved that given $n$ bodies with positive equal masses at the vertices of a regular $n$-gon the body $n+1$ must belong to an axis of symmetry of the $n$-gon in order that the $n+1$ bodies perform a central configuration. In this context we have the following result for the planar central configurations.

Theorem 1. Consider $n \geq 2$ bodies with positive equal masses $m=m_{1}=\cdots=m_{n}$ and position vectors $r_{1}, \cdots, r_{n}$ at the vertices of a regular $n-g o n$, and an $n+1$ body of null mass. The following statements hold.

(a) There are 5 classes of central configurations for the $(n+1)$-body problem when $n=2$, see Figure 1(a).

(b) There are $3 n+1$ classes of central configurations for the $(n+1)$-body problem when $n \geq 3$, see Figures 1(b) and 2 for $n=3$ and $n=4$, respectively.

In [5] Bang and Elmabsout studied the following slightly variation of the problem here studied. Consider $(n+1)+1$ bodies in a plane where $n$ bodies with positive equal masses are at the vertices of a regular $n$-gon and one body with mass $m_{0} \geq 0$ is at the center of the $n$-gon. Where can a body with null mass be located in order to have a new central configuration? Bang and Elmabsout solved completely the above problem by using their previous result in [4]. We emphasize that the problem studied in [5] is slightly different from the one studied here. Nevertheless the proof of Proposition 1, page 306 of [5], will be adapted to prove statement (b) of our Theorem 1.

Little is known about the spatial central configurations. Cedó and Llibre [6] proved that the regular polyhedra with equal masses at their vertices are spatial central configurations. 


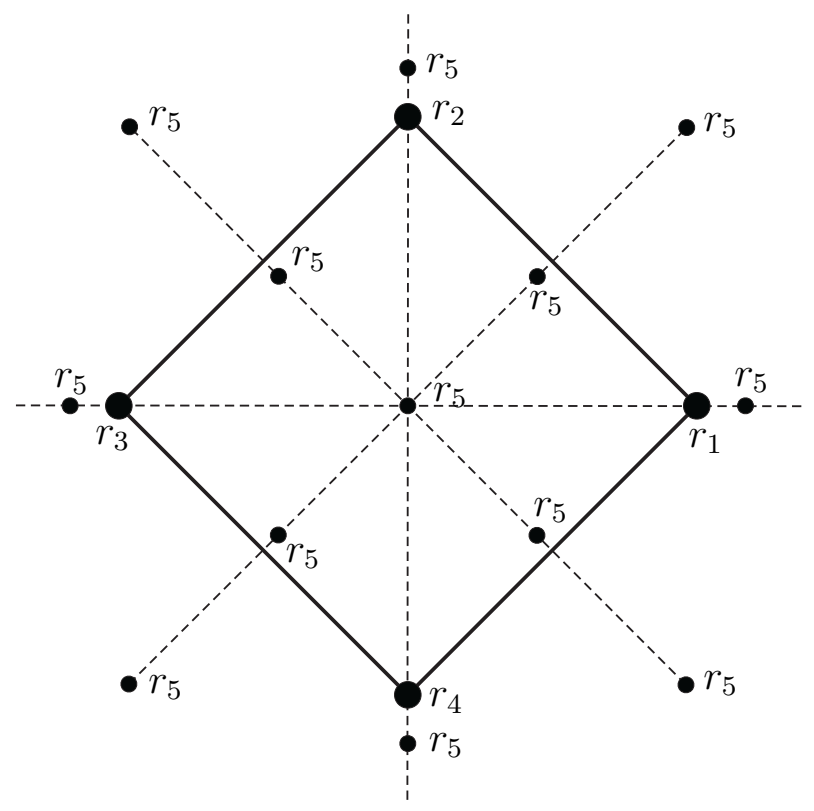

FIgURE 2. Four bodies with equal masses and position vectors $r_{1}, r_{2}, r_{3}, r_{4}$ at the vertices of a square and the 13 possible positions $r_{5}$ for the fifth body with null mass.

Santos and Vidal [20, 21] proved the existence of 25 possible positions for a body with null mass when 4 bodies with equal masses are at the vertices of a regular tetrahedron. As in the planar case, these positions are on the straight lines of symmetry of the tetrahedron.

With the following result we complete the study of the initial problem in the case where the bodies with equal masses are at the vertices of a regular polyhedron.

Theorem 2. Consider $n$ bodies with positive equal masses $m=m_{1}=\cdots=m_{n}$ and position vectors $r_{1}, \cdots, r_{n}$ at the vertices of a regular polyhedron, and an $n+1$ body of null mass. Assuming that in order to have a central configuration the only possible positions for the body of null mass are on the axes of symmetry of the regular polyhedra the following statements hold.

(a) There are 45 classes of central configurations for the $(n+1)$-body problem when $n=8$, that is when the regular polyhedron is the cube.

(b) There are 47 classes of central configurations for the $(n+1)$-body problem when $n=6$, that is when the regular polyhedron is the octahedron.

(c) There are 105 classes of central configurations for the $(n+1)$-body problem when $n=20$, that is when the regular polyhedron is the dodecahedron.

(d) There are 113 classes of central configurations for the $(n+1)$-body problem when $n=12$, that is when the regular polyhedron is the icosahedron.

We conjecture that the assumption done in the statement of Theorem 2: In order to have a central configuration of the $(n+1)$-body problem when $n$ bodies of equal mass are at the vertices of a regular polyhedra, the only possible positions for the $n+1$ body of null mass are on the axes of symmetry of the regular polyhedra, is redundant; i.e. there are no central 
configurations of this $(n+1)$-body problem if the $n+1$ body of null mass is not on one of these axes of symmetry.

This article is organized as follows. Theorem 1 is proved in section 2 and Theorem 2 is proved in section 3 .

\section{Proof of Theorem 1}

From the equations of central configurations (1) we write

$$
f_{i}=\lambda r_{i}+\sum_{\substack{j=1 \\ j \neq i}}^{n} \frac{m_{j}}{r_{i j}^{3}}\left(r_{i}-r_{j}\right)=0,
$$

for $i=1, \ldots, n$.

We start proving statement (a) of Theorem 1. Consider two bodies with masses $m=$ $m_{1}=m_{2}>0$ at $r_{1}=(1,0)$ and $r_{2}=(-1,0)$. From equations $f_{1}=0$ and $f_{2}=0$ of (2) we obtain $\lambda=-m / 4$. Substituting this value into the equations $f_{i}=0$ we obtain

$$
-\frac{x}{4}+\frac{x+1}{\left((x+1)^{2}+y^{2}\right)^{3 / 2}}+\frac{x-1}{\left((x-1)^{2}+y^{2}\right)^{3 / 2}}=0
$$

and

$$
y\left[-\frac{1}{4}+\frac{1}{\left((x+1)^{2}+y^{2}\right)^{3 / 2}}+\frac{1}{\left((x-1)^{2}+y^{2}\right)^{3 / 2}}\right]=0 .
$$

From equation (4) we conclude that either $y=0$, or

$$
\frac{1}{\left((x+1)^{2}+y^{2}\right)^{3 / 2}}+\frac{1}{\left((x-1)^{2}+y^{2}\right)^{3 / 2}}=\frac{1}{4} \text {. }
$$

Substituting $y=0$ into equation (3) we obtain

$$
-\frac{x}{4}+\frac{x+1}{\left((x+1)^{2}\right)^{3 / 2}}+\frac{x-1}{\left((x-1)^{2}\right)^{3 / 2}}=0,
$$

whose solutions are $x=0$ and $x= \pm \alpha= \pm 2.39681$ with five decimal round-off coordinates. Now, from equations (3) and (5), by a straightforward calculation we obtain $x=0$ and $y= \pm \sqrt{3}$. In short, when $n=2$ we have the following five possibilities for $r_{3}$. This completes the proof of statement (a) of Theorem 1 (see Figure 1 (a)):

$$
r_{3}=(0,0), \quad r_{3}=( \pm \alpha, 0), \quad r_{3}=(0, \pm \sqrt{3}) .
$$

Of course, these five central configurations corresponds to the five central configurations of the 3-body problem, founded by Euler and Lagrange, when one of the three masses is zero.

Instead of equations (2), consider the following equivalent set of equations, called (planar) Dziobek-Laura-Andoyer equations (see [7, 11]),

$$
f_{i, j}=\sum_{\substack{k=1 \\ k \neq i, j}}^{n} m_{k}\left(R_{i k}-R_{j k}\right) \Delta_{i j k}=0,
$$


for $1 \leq i<j \leq n$, where $R_{i j}=1 / r_{i j}^{3}$ and $\Delta_{i j k}=\left(r_{i}-r_{j}\right) \wedge\left(r_{i}-r_{k}\right)$. Note that $\Delta_{i j k}$ is twice the oriented area of the triangle formed by the bodies at $r_{i}, r_{j}$ and $r_{k}$. This set of equations is formed by $n(n-1) / 2$ equations.

Without loss of generality we take the $n$ bodies with equal masses at the vertices of a regular $n$-gon labeled counterclockwise and such that $r_{1}=(1,0)$, see Figures $1(\mathrm{~b})$ and 2. We separate the proof according to the parity of $n$.

When $n$ is odd the axes of symmetry of the $n$-gon are determined by the straight lines passing through a vertex and the center of the $n$-gon. By the symmetries of the problem proved in [4], it is sufficient to study the equation $f_{n, n+1}=0$ under the assumption $y=0$. By a similar analysis given in the proof of Proposition 1, page 306 of [5], we obtain the following four possibilities for $r_{n+1}$ (see Figure 1 (b)):

$$
r_{n+1}=(0,0), \quad r_{n+1}=\left(\rho_{1}, 0\right), \quad r_{n+1}=\left(-\rho_{2}, 0\right), \quad r_{n+1}=\left(-\rho_{3}, 0\right),
$$

with $\rho_{1}>1$, and $\rho_{2}, \rho_{3}>0$. So, clearly when $n$ is odd we get $3 n+1$ central configurations for the $(n+1)$-body problem.

Assume $n>2$ is even, then the axes of symmetry of the $n$-gon are determined by either the straight lines passing through a vertex and the center of the $n$-gon (the diagonals), or by the mediatrices of the sides of the $n$-gon. For the first case it is enough, by the symmetries of the problem proved in [4], to study only the equation $f_{n, n+1}=0$ under the assumption $y=0$. Again, we obtain the following three possibilities for $r_{n+1}$ (see Figure 2):

$$
r_{n+1}=(0,0), \quad r_{n+1}=\left( \pm \rho_{2}, 0\right), \quad \rho_{2}>1 .
$$

For the second case it is enough to study the equation $f_{n, n+1}=0$ under the assumption $y=\tan (\pi / n) x$. We obtain the following five possibilities for $r_{n+1}$ (see Figure 2):

$$
r_{n+1}=(0,0), \quad r_{n+1}= \pm \rho_{3}\left(1, \tan \left(\frac{\pi}{n}\right)\right), \quad r_{n+1}= \pm \rho_{4}\left(1, \tan \left(\frac{\pi}{n}\right)\right),
$$

with $0<\rho_{3}<1$, and $\rho_{4}>1$.

In short if $n=2 k$, then we have $k$ diagonals and $k$ mediatrices for the $n$-gon. For each diagonal we have two central configurations, and for each mediatrix we have four central configurations without counting the central configuration with the null mass at the origin of coordinates. Therefore we have $2 k+4 k+1=6 k+1=3(2 k)+1=3 n+1$ central configurations. Hence Theorem 1 is proved.

\section{Proof of TheOREM 2}

For the proof of Theorem 2 we use (spatial) Dziobek-Laura-Andoyer equations (see [13]). These $n(n-1)(n-2) / 2$ equations are equivalent to the equations in (2) and are given by

$$
f_{i, j, h}=\sum_{\substack{k=1 \\ k \neq i, j, h}}^{n} m_{k}\left(R_{i k}-R_{j k}\right) \Delta_{i j h k}=0,
$$

for $1 \leq i<j \leq n, h=1, \ldots, n, h \neq i, j$. In (6) we have $R_{i j}=1 / r_{i j}^{3}$ and $\Delta_{i j h k}=$ $\left(r_{i}-r_{j}\right) \wedge\left(r_{j}-r_{h}\right) \cdot\left(r_{h}-r_{k}\right)$, which is six times the signed volume of the tetrahedron with vertices in the bodies localized at $r_{i}, r_{j}, r_{h}$ and $r_{k}$. 
In what follows we will prove that if the body with null mass is at suitable positions on an axis of symmetry of a regular polyhedron, then the configuration performs a central configuration for the spatial restricted $(n+1)$-body problem.

Proof of statement (a) Theorem 2. The hypotheses that we concern in this case is that the eight bodies with positive equal masses are disposed at the vertices of a cube. Without loss of generality we consider the following coordinates for those vertices

$$
\begin{aligned}
& r_{1}=(1,1,-1), \quad r_{2}=(1,1,1), \quad r_{3}=(1,-1,1), \quad r_{4}=(1,-1,-1), \\
& r_{5}=(-1,1,-1), \quad r_{6}=(-1,1,1), \quad r_{7}=(-1,-1,1), \quad r_{8}=(-1,-1,-1) .
\end{aligned}
$$

We also assume masses $m_{i}=1, i \in\{1, \ldots, 8\}$, for the bodies at the vertices of the cube and $m_{9}=0$ for the ninth body with the position vector $r_{9}=(x, y, z)$.

Since the spatial configuration of eight bodies with equal masses at the vertices of a cube is a well known central configuration, and by assumptions the position of the null mass must be on some of the axes of symmetry of the cube, after some straightforward calculations with the equations $(6)$ of the restricted $(8+1)$-body problem, they are either trivially satisfied or equivalent to only one equation. We will analyze this remaining equation in each of the three types of symmetry axes of the cube.

Case 1. The first class of symmetry contains 3 straight lines each of them passing through the centers of two opposite faces. Without loss of generality we consider the ninth body belonging to the straight line $y=0$ and $z=0$. In this case the remaining equation is

$$
\begin{aligned}
f_{8,9,7}= & \frac{8}{((x-2) x+3)^{3 / 2}}-\frac{8}{(x(x+2)+3)^{3 / 2}}+ \\
& +x\left(-\frac{8}{((x-2) x+3)^{3 / 2}}-\frac{8}{(x(x+2)+3)^{3 / 2}}+\frac{1}{36}(18+9 \sqrt{2}+2 \sqrt{3})\right)=0 .
\end{aligned}
$$

We can easily compute the solutions of this equation and we get

$$
x=0, \quad x= \pm 0.89328 \text { and } x= \pm 2.20832,
$$

with five decimal round-off coordinates. Thus, in this case there are 13 positions for the ninth body in order to have a central configuration.

Case 2. The second class of symmetry contains 6 straight lines each of them passing through the middle points of opposite edges with respect to the origin of coordinates. Without loss of generality we consider the ninth body belonging to the line $y=x$ and $z=0$. In this case we obtain the remaining equation

$$
\begin{aligned}
f_{8,9,6}= & \frac{8 x}{\left(2 x^{2}+3\right)^{3 / 2}}-\frac{1}{36}(18+9 \sqrt{2}+2 \sqrt{3}) x+\frac{4 x}{(2(x-2) x+3)^{3 / 2}}+ \\
& +\frac{4 x}{(2 x(x+2)+3)^{3 / 2}}-\frac{4}{(2(x-2) x+3)^{3 / 2}}+\frac{4}{(2 x(x+2)+3)^{3 / 2}}=0 .
\end{aligned}
$$

The solutions of this equation are

$$
x=0, \quad x= \pm 0.97667 \text { and } x= \pm 1.74249,
$$

with five decimal round-off coordinates. Therefore we have 24 new positions for the ninth body in order to have a central configuration. 
Case 3. This class of symmetry contains 4 straight lines each of them being a diagonal of the cube passing through opposite vertices with respect to the origin of coordinates. Without loss of generality we consider the ninth body belonging to the line $x=y=z$. In this case, the remaining equation is

$$
\begin{aligned}
f_{7,9,6}= & -\frac{8 \sqrt{3}(x-1)}{|x-1|^{3}}-\frac{72}{\left(3 x^{2}+2 x+3\right)^{3 / 2}}+\frac{72}{\left(3 x^{2}-2 x+3\right)^{3 / 2}}-\frac{8 \sqrt{3}}{(x+1)^{3}}+ \\
& +x\left(-\frac{216}{\left(3 x^{2}+2 x+3\right)^{3 / 2}}-\frac{216}{\left(3 x^{2}-2 x+3\right)^{3 / 2}}-\frac{8 \sqrt{3}}{(x+1)^{3}}+9 \sqrt{2}+2 \sqrt{3}+18\right)=0 .
\end{aligned}
$$

The solutions of this equation are

$$
x=0, \quad x= \pm 1.64365,
$$

with five decimal round-off coordinates. Thus we get 8 new positions for the ninth body in order to have a central configuration.

In short, if the ninth body with null mass is either at the origin or at one of the 44 positions calculated above the nine bodies are in a central configuration. Therefore statement (a) Theorem 2 is proved.

Proof of statement (b) Theorem 2. Now we are considering six bodies with positive equal masses at the vertices of an octahedron. Without loss of generality we consider the following coordinates

$$
\begin{array}{lll}
r_{1}=(1,1,0), & r_{2}=(-1,1,0), & r_{3}=(-1,-1,0), \\
r_{4}=(1,-1,0), & r_{5}=(0,0, \sqrt{2}), & r_{6}=(0,0,-\sqrt{2}),
\end{array}
$$

the masses $m_{i}=1, i \in\{1, \ldots, 6\}$, for the bodies at the vertices of the octahedron and $m_{7}=0$ for the seventh body with the position vector $r_{7}=(x, y, z)$.

Again, under the assumptions of Theorem 2, it results that the equations (6) for this restricted $(6+1)$-body problem are either trivially satisfied or equivalent to only one equation. We will analyze this remaining equation in each of the three types of axes of symmetry of the octahedron.

Case 1. The first class of symmetry contains 3 straight lines each of them passing through two opposite vertices with respect to the origin of coordinates. Without loss of generality we consider the seventh body belonging to the straight line $x=0$ and $y=0$. In this case we obtain the remaining equation

$$
f_{1,7,2}=\frac{8 z}{\left(z^{2}+2\right)^{3 / 2}}-\frac{1}{8}(8+\sqrt{2}) z-\frac{2 \sqrt{(\sqrt{2}-z)^{2}}}{(\sqrt{2}-z)^{3}}+\frac{2 \sqrt{(z+\sqrt{2})^{2}}}{(z+\sqrt{2})^{3}}=0 .
$$

The solutions of this equation are

$$
z=0 \text { and } z= \pm 2.44638,
$$

with five decimal round-off coordinates. Thus there are 7 positions for the seventh body in order to have a central configuration.

Case 2. The second class of symmetry contains 6 straight lines each of them passing through the middle points of two opposite edges with respect to the origin of coordinates. Without 
loss of generality we consider the seventh body belonging to the line $y=0$ and $z=0$. In this case we obtain the remaining equation

$$
\begin{aligned}
f_{1,7,5}= & 2 \sqrt{2}\left(\frac{1}{\left(x^{2}+2 x+2\right)^{3 / 2}}-\frac{1}{\left(x^{2}-2 x+2\right)^{3 / 2}}\right)+ \\
& +x\left(\frac{2 \sqrt{2}}{\left(x^{2}-2 x+2\right)^{3 / 2}}+\frac{2 \sqrt{2}}{\left(x^{2}+2 x+2\right)^{3 / 2}}+\frac{2 \sqrt{2}}{\left(x^{2}+2\right)^{3 / 2}}-\frac{1}{\sqrt{2}}-\frac{1}{8}\right)=0 .
\end{aligned}
$$

By a straightforward calculation the solutions of this equation are

$$
x=0, \quad x= \pm 0.86349 \text { and } x= \pm 1.99344
$$

with five decimal round-off coordinates. Hence we have 24 new positions for the seventh body in order to have a central configuration.

Case 3. This class of symmetry contains 4 straight lines each of them passing through the centers of two opposite faces. Without loss of generality we consider the seventh body belonging to the line $x=0, y=2 t / 3$ and $z=t \sqrt{2} / 3, t \in \mathbb{R}$. In this case, we obtain the remaining equation

$$
\begin{aligned}
f_{1,7,2}= & 3 \sqrt{3}\left(\frac{1}{(t(t+2)+3)^{3 / 2}}-\frac{1}{((t-2) t+3)^{3 / 2}}\right)+ \\
& +t\left(\frac{3 \sqrt{3}}{(t(t+2)+3)^{3 / 2}}+\frac{3 \sqrt{3}}{((t-2) t+3)^{3 / 2}}-\frac{\sqrt{2}}{3}-\frac{1}{12}\right)=0 .
\end{aligned}
$$

By a straightforward calculation the solutions of this equation are

$$
t=0, \quad t= \pm 0.82676 \text { and } t= \pm 2.34709 \text {, }
$$

with five decimal round-off coordinates. Then we get 16 new positions for the seventh body in order to have a central configuration.

By the above analyzes, if the seventh body with null mass is either at the origin or at one of the 46 positions calculated in cases 1, 2 and 3 the seven bodies are in a central configuration. Thus statement (b) of Theorem 2 is proved.

Proof of statement (c) Theorem 2. Consider twenty bodies with positive equal masses at the vertices of a dodecahedron. Without loss of generality we can take the following coordinates for those vertices

$$
\begin{array}{llll}
r_{1}=(1,1,1), & r_{2}=(-1,1,1), & r_{3}=(1,-1,1), & r_{4}=(1,1,-1) \\
r_{5}=(-1,-1,1), & r_{6}=(-1,1,-1), & r_{7}=(1,-1,-1), & r_{8}=(-1,-1,-1) \\
r_{9}=(0, \phi, 1 / \phi), & r_{10}=(0, \phi,-1 / \phi), & r_{11}=(0,-\phi, 1 / \phi), & r_{12}=(0,-\phi,-1 / \phi) \\
r_{13}=(1 / \phi, 0, \phi), & r_{14}=(1 / \phi, 0,-\phi), & r_{15}=(-1 / \phi, 0, \phi), & r_{16}=(-1 / \phi, 0,-\phi) \\
r_{17}=(\phi, 1 / \phi, 0), & r_{18}=(\phi,-1 / \phi, 0), & r_{19}=(-\phi, 1 / \phi, 0), & r_{20}=(-\phi,-1 / \phi, 0),
\end{array}
$$

where $\phi=(1+\sqrt{5}) / 2$, the masses $m_{i}=1, i \in\{1, \ldots, 20\}$, for the bodies at the vertices of the dodecahedron and $m_{21}=0$ for the twenty-first body with the position vector $r_{21}=(x, y, z)$.

Under the assumptions of Theorem 2 the equations (6) of this restricted $(20+1)$-body problem are either trivially satisfied or equivalent to only one equation. We will analyze this remaining equation in each of the three types of axes of symmetry of the dodecahedron. 
Case 1. The first class of symmetry contains 10 straight lines each of them passing through two opposite vertices with respect to the origin of coordinates. Without loss of generality we consider the twenty-first body belonging to the straight line $x=y=z$. In this case, we obtain the remaining equation $f_{2,21,3}=0$. We will omit the expression of $f_{2,21,3}$ since it is too long to be printed. The solutions of this equation are

$$
x=0 \quad \text { and } \quad x= \pm 1.46222,
$$

with five decimal round-off coordinates. Thus there are 21 positions for the twenty-first body in order to have a central configuration.

Case 2. The second class of symmetry contains 15 straight lines each of them passing through the middle points of two opposite edges with respect to the origin of coordinates. Without loss of generality we consider the twenty-first body belonging to the line $y=0$ and $z=0$. In this case, we obtain the remaining equation $f_{1,21,3}=0$. The expression of $f_{1,21,3}$ will be omitted becuase it is too long to be printed. The solutions of this equation are

$$
x=0, \quad x= \pm 1.63543 \text { and } x= \pm 2.38786,
$$

with five decimal round-off coordinates. Then we have 60 new possible positions for the twenty-first body in order to have a central configuration.

Case 3. This class of symmetry contains 6 straight lines each of them passing through the centers of two opposite faces with respect to the origin of coordinates. Without loss of generality we consider the twenty-first body belonging to the line $x=s(5+3 \sqrt{5}) / 10, y=0$ and $z=s(5+\sqrt{5}) / 10, s \in \mathbb{R}$. In this case we obtain the remaining equation $f_{2,21,2}=0$. Again the expression of $f_{2,21,2}$ will be omitted because it is too long to be printed. The solutions of this equation are

$$
s=0, \quad s= \pm 1.06245 \text { and } s= \pm 1.56090,
$$

with five decimal round-off coordinates. So we obtain 24 new possible positions for the twenty-first body in order to have a central configuration. Therefore if the twenty-first body with null mass is either at the origin or at one of the 104 positions calculated in cases 1,2 and 3 the twenty one body is in a central configuration. The proof of statement (c) Theorem 2 is completed.

Proof of statement (d) Theorem 2. Now we are considering twelve bodies with positive equal masses at the vertices of an icosahedron. Without loss of generality we consider the following coordinates for those vertices

$$
\begin{aligned}
& r_{1}=(0,1, \phi), \quad r_{2}=(0,1,-\phi), \quad r_{3}=(0,-1, \phi), \quad r_{4}=(0,-1,-\phi) \\
& r_{5}=(1, \phi, 0), \quad r_{6}=(1,-\phi, 0), \quad r_{7}=(-1, \phi, 0), \quad r_{8}=(-1,-\phi, 0) \\
& r_{9}=(\phi, 0,1), \quad r_{10}=(\phi, 0,-1), \quad r_{11}=(-\phi, 0,1), \quad r_{12}=(-\phi, 0,-1) \text {, }
\end{aligned}
$$

where $\phi=(1+\sqrt{5}) / 2$, the masses $m_{i}=1, i \in\{1, \ldots, 12\}$, for the bodies at the vertices of the icosahedron and $m_{13}=0$ for the thirteenth body with the position vector $r_{13}=(x, y, z)$.

The equations $(6)$ of this restricted $(12+1)$-body problem are either trivially satisfied or equivalent to only one equation. We will analyze the remaining equation in each of the three types of axes of symmetry of the icosahedron. 
Case 1. The first class of symmetry contains 6 straight lines each of them passing through two opposite vertices with respect the origin of coordinates. Without loss of generality consider the thirteenth body belonging to the line $x=t(1+\sqrt{5}) / 2, y=0, z=-t, t \in \mathbb{R}$. The remaining equation is $f_{12,13,7}=0$. We will omit the expression of $f_{12,13,7}$ because it is too long to be printed. The solutions of this equation are

$$
t=0 \text { and } t= \pm 1.54935 \text {, }
$$

with five decimal round-off coordinates. Thus there are 13 positions for the thirteenth body in order to have a central configuration.

Case 2. The second class of symmetry contains 15 straight lines each of them passing through the middle points of two opposite edges with respect to the origin of coordinates. Without loss of generality we consider the thirteenth body belonging to the line $x=s(3+$ $\sqrt{5}) / 4, y=s(-1-\sqrt{5}) / 4$ and $z=-s / 2$ with $s \in \mathbb{R}$. In this case we obtain the remaining equation $f_{12,13,7}=0$. Again the expression of $f_{12,13,7}$ will be omitted becuase it is too long to be printed. The solutions of the above equation are

$$
s=0, \quad s= \pm 0.98408 \text { and } s= \pm 1.59109
$$

with five decimal round-off coordinates. Thus we have 60 new positions for the thirteenth body in order to have a central configuration.

Case 3. This class of symmetry contains 10 straight lines each of them passing through the centers of two opposite faces with respect to the origin of coordinates. Without loss of generality we consider the thirteenth body belonging to the line $x=\alpha(3+\sqrt{5}) / 6, y=$ $\alpha(-3-\sqrt{5}) / 6$ and $z=\alpha(-3-\sqrt{5}) / 6$ with $\alpha \in \mathbb{R}$. In this case we obtain the remaining equation $f_{12,13,7}=0$. Again the expression of $f_{12,13,7}$ will be omitted because it is too long to be printed. The solutions of this equation are

$$
\alpha=0, \quad \alpha= \pm 1.00082 \quad \text { and } \quad \alpha= \pm 1.66419
$$

with five decimal round-off coordinates. So we obtain 40 new positions for the thirteenth body in order to have a central configuration.

From the above analysis if the thirteenth body with null mass is either at the origin or at one of the 112 positions calculated in cases 1,2 and 3 the thirteen body is in a central configuration. The proof of statement (d) of Theorem 2 is done.

\section{ACKNOWLEDGEMENTS.}

The first, second and fourth authors are partially supported by Fundação de Amparo à Pesquisa do Estado de Minas Gerais (FAPEMIG) grant APQ-001082/14. The third author is partially supported by the MINECO grants MTM2013-40998-P and MTM2016-77278-P (FEDER) and the AGAUR grant 2014 SGR568. The fourth author is partially supported by Capes/Estágio Sênior no Exterior grant number 88881.119020/2016-01. In the course of this work the fourth author was a visitor at Texas Christian University and gratefully acknowledges its warm hospitality. 


\section{REFERENCES}

[1] A. Albouy, A. Chenciner, Le problème des n corps et les distances mutuelles, Invent. math. 131 (1998), 151-184.

[2] A. Albouy, V. Kaloshin, Finiteness of central configurations of five bodies in the plane, Annals of Math. 176 (2012), 535-588.

[3] R.F. Arenstorf, Central configurations of four bodies with one inferior mass, Celestial Mech. 28 (1982), 9-15.

[4] D. Bang, B. Elmabsout, Representations of complex functions, means on the regular $n-g o n$ and applications to gravitational potential, J. Phys. A 36 (2003), 11435-11450.

[5] D. Bang, B. Elmabsout, Restricted $N+1$-body problem: existence and stability of relative equilibria, Celestial Mech. Dynam. Astronom. 89 (2004), 305-318.

[6] F. Cedó, J. LliBRE, Symmetric central configurations of the spatial n-body problem, J. of Geometry and Physics 6 (1989), 367-394.

[7] O. DzIOBEK, Ueber einen merkwürdigen Fall des Vielkörperproblems, Astron. Nach. 152 (1900), 32-46.

[8] L. Euler, De moto rectilineo trium corporum se mutuo attahentium, Novi Comm. Acad. Sci. Imp. Petrop. 11 (1767), 144-151.

[9] G. Gómez, J. Llibre, R. Martínez, C. Simó, Dynamics and Mission Design Near Libration Points. Vol. I Fundamentals: The case of collinear libration points. World Scientific Monograph Series in Mathematics, Vol. 2, World Scientific, Singapore, 2001.

[10] G. Gómez, J. Llibre, R. Martínez, C. Simó, Dynamics and Mission Design Near Libration Points. Vol. II Fundamentals: The case of triangular libration points. World Scientific Monograph Series in Mathematics. Vol. 3, World Scientific, Singapore, 2001.

[11] Y. Hagihara, Celestial Mechanics, vol. 1. Cambridge, Massachusetts, USA, MIT Press 1970.

[12] H. Hampton, R. Moeckel, Finiteness of relative equilibria of the four-body problem, Inventiones math. 163 (2006), 289-312.

[13] M. Hampton, M. Santoprete, Seven-body central configurations: a family of central configurations in the spatial seven-body problem, Celestial Mech. Dyn. Astr. 99 (2007), 293-305.

[14] J.L. Lagrange, Essai sur le problème de trois corps, Euvres, vol. 6, Gauthier-Villars, Paris, 1873.

[15] K.R. MEYER, Bifurcation of a central configuration, Cel. Mech. 40 (1987), 273-282.

[16] R. Moeckel, On central configurations, Mah. Z. 205 (1990), 499-517.

[17] J.I. Palmore, Classifying relative equilibria II, Bull. Amer. Math. Soc. 81 (1975), 71-73.

[18] P. Pedersen, Librationspunkte im restringierten Vierkörperproblem, Danske Vid. Selsk. Math.-Fys. 21 (1944), $80 \mathrm{pp}$.

[19] D. SAARI, On the role and properties of central configurations, Cel. Mech. 21 (1980), 9-20.

[20] A.A. SAntos, Dziobek's configurations in restricted problems and bifurcation, Celestial Mech. Dynam. Astronom. 90 (2004), 213-238.

[21] A.A. Santos, C. Vidal, Symmetry of the restricted $4+1$-body problem with equal masses, Regular and Chaotic Dynamics 12 (2007), 27-38.

[22] D.S. Schmidt, Central configurations in $\mathbb{R}^{2}$ and $\mathbb{R}^{3}$, Contemporary Math. 81 (1980), 59-76.

[23] S. Smale, Topology and mechanics II: The planar $n$-body problem, Inventiones math. 11 (1970), 45-64.

[24] A. Wintner, The Analytical Foundations of Celestial Mechanics, Princeton University Press, 1941.

[25] Z. XIA, Central configurations with many small masses, J. Differential Equations 91 (1991), $168-179$.

${ }^{A}$ Instituto de Matemática e Computação, Universidade Federal de Itajubá, Avenida BPS 1303, Pinheirinho, CEP 37.500-903, ItajubÁ, MG, Brazil

E-mail address: acfernandes@unifei.edu.br, braulio@unifei.edu.br, lfmelo@unifei.edu.br

${ }^{B}$ Departament de Matemàtiques. Universitat Autònoma de Barcelona, Bellaterra, 08193Barcelona, Catalonia, Spain

E-mail address: jllibre@mat.uab.cat 